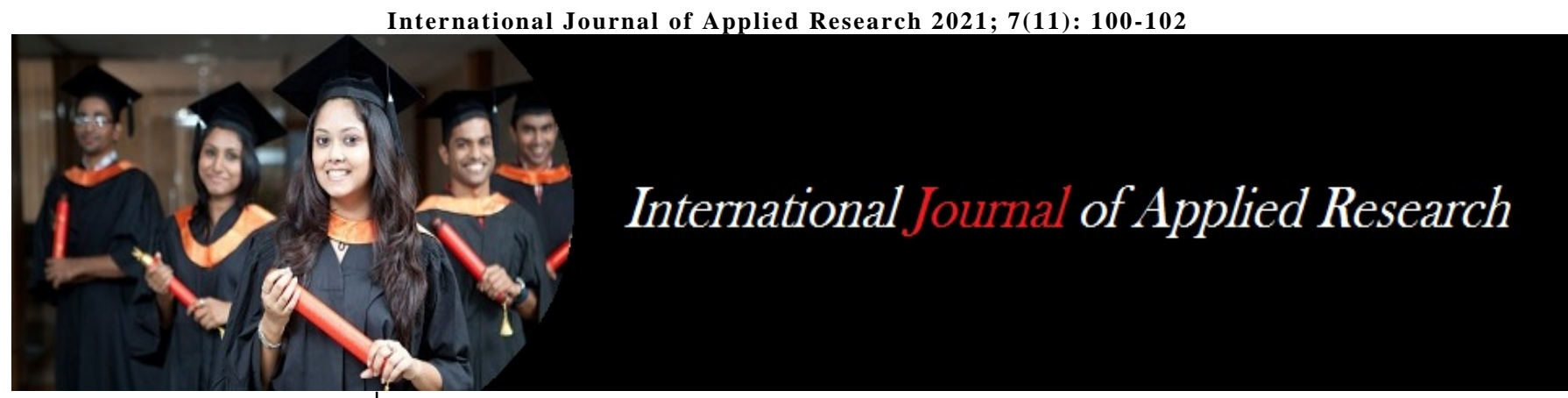

ISSN Print: 2394-7500 ISSN Online: 2394-5869 Impact Factor: 8.4 IJAR 2021; 7(11): 100-102 www.allresearchjournal.com

Received: 12-09-2021 Accepted: 14-10-2021

Ihor Korol

PA-C, MPAS, Physician

Assistant in an Outpatient

Military Primary Care Clinic.

Killeen, Texas, USA

Ksenia Strakhova

MSDH, Dental Hygienist in a

Community Dental Clinic.

Killeen, Texas, USA

Matheus Azevedo

AS, Emergency Medical

Technician in an outpatient military primary care clinic,

Killeen, Texas, USA

Robin H. Baumeisterm

MS, Freelance Biomedical Anthropologist. Killeen, Texas, USA
Corresponding Author: Ihor Korol

PA-C, MPAS, Physician Assistant in an outpatient Military Primary care clinic.

Killeen, Texas, USA

\section{Battlefield acupuncture: Effectiveness for treatment of acute and subacute pain in a military primary care setting}

\author{
Ihor Korol, Ksenia Strakhova, Matheus Azevedo and Robin H. \\ Baumeisterm
}

DOI: $\underline{\text { https://doi.org/10.22271/allresearch.2021.v7.i11b.9108 }}$

\section{Abstract}

Objective: Battlefield Acupuncture (BFA) is a form of auricular acupuncture that is increasingly rising in popularity as a method of managing pain in military settings. This study aims to contribute to the growing body of literature on the efficacy of BFA by evaluating pain pre- and post- BFA administration and by measuring that pain by level and type.

Materials and Methods: This was a retrospective study evaluating active duty Army members who were receiving care at the outpatient military clinic. Data evaluated all patients undergoing a BFA procedure between March 1, 2020, and November 1, 2020. The pain was rated using a standardized DOD/VA pain scale.

Results: Of the 26 patients that met our inclusion criteria, 21 (81\%) experienced a decrease in pain following BFA administration, while 5 (19\%) experienced no change. No patients experienced an increase in pain. Individuals with a baseline pain of 6 or less experienced a greater decrease in pain than did those with a baseline pain of more than 6 . Those with low back pain also experienced a greater reduction in pain than did those with other pain types.

Conclusions: BFA effectively reduces pain and can be considered a promising treatment option for pain reduction in a military setting. Further research is needed to evaluate the efficacy of BFA on various pain types.

Keywords: Battlefield, Effectiveness, military primary, Subacute pain

\section{Introduction}

The reduction and management of pain are of critical importance in within a military context. Due to the high risk of injury and the need for quick recovery, medications that foster adverse effects should be avoided ${ }^{[1]}$. The revaluation of current treatment options as compared to novel developments can prove beneficial, as novel methods have the potential to increase efficacy while reducing risk.

The most common treatments for acute and subacute pain are pharmacological. Of these pharmacological treatment options, opioids are the riskiest; while effective, they are host to several detrimental effects and outcomes for a patient, including addiction, heightened pain sensitivity, and the possibility of addiction-related death ${ }^{[2]}$. Especially as opioid misuse and mortality rates increase, it is becoming critically more important to phase them out of standard practice. Nonsteroidal anti-inflammatory drugs (NSAIDs) —including Ketorolac, our current standard of care-are common alternatives to opioids for the management of pain due to their milder side effect profile; however, they also have their cons of variable efficacy, limited dosage, and high cardiovascular or bleeding risk, especially gastrointestinal ${ }^{[3]}$.

Due to the extensive side effect profiles, limited efficacy, and short span of the possible use of NSAIDs, opioids, and other prescription and over-the-counter drugs, non-pharmacological pain management options have rapidly increased in popularity ${ }^{[4]}$. Battlefield acupuncture (BFA) is one such rising alternative to pharmacological pain management. BFA, adapted from traditional Chinese medicine, was developed in 2001 by Richard Niemtzow as a safe and cost-effective alternative to pharmacological methods and BFA can be easily trained in a military setting ${ }^{[5]}$. As a form of auriculotherapy (ear acupuncture), BFA is practiced by placing semi-permanent gold needles into specific sites on one or both ears ${ }^{[6]}$. 
There is some support for BFA in reducing pain and insomnia and combining BFA and standard post-surgical care to manage post-surgical pain ${ }^{[7,1]}$. Despite these studies, the efficacy of BFA is still under consideration, and supportive evidence is limited and inconsistent $[8,9]$. However, its relative cost-effectiveness, longevity, and reduction of harmful outcomes render it a relevant subject of study were efficacy to be confirmed ${ }^{[8]}$.

Acupuncture has found a place in military settings for thousands of years but was not considered in the U.S. Armed Forces until the 1980s and was not used on a widespread scale until the 1990 s $^{[10]}$. Its great relevance to a military setting is founded in its low-cost and low-risk modality; in a context where the risk for pain is as high as the need for a rapid recovery rate, having pain relief modalities in place that can facilitate high efficacy of pain relief in a short amount of time without the risk of adverse effects is critical ${ }^{[10]}$. Because BFA is psychobiological in the mechanism, it does not incur any risks associated with pharmacological painkillers, and further does not maintain any limitations on dosage or repeat administration that are great hindrances with NSAIDs, opioids, and other pharmacological agents ${ }^{[6,10]}$.

This study aims to evaluate the practice of BFA as an alternative to prescription drugs, such as Ketorolac. Testing the efficacy of non-pharmacological pain management techniques can lead to the revaluation of the standard of care if BFA proves to be equally or more effective than Ketorolac, or if their combination proves most effective. Finding the most efficacious and safest pain management methods can improve patient outcomes, shorten recovery times, and allow for a quicker return to active duty. Were BFA to prove beneficial in either isolation or combination, BFA training should be advocated for.

\section{Materials and Methods}

The population selected for evaluation in this retrospective study was active duty Army members assigned to the outpatient military clinic within United States, (a population of approx. 7000 patients) from ages 17 to 62 with an onset of pain within 30 days or less at the time of the intervention. Exclusion criteria were pregnant women, those with a dermatologic condition of the external auricle or the presence of an otolaryngology disease of external ears, relocation, separation from military service within six months of intervention, or an allergy to NSAIDs. Screening for pregnancy was conducted verbally according to the checklist used to assess pregnancy's possibility based on recommendations by Curtis et al. ${ }^{[11]}$.

The data for this retrospective case-control study were collected during routine primary care evaluations of all subjects who underwent a BFA procedure between March 1, 2020, and November 1, 2020. If BFA was an option of treatment for a patient's condition, it was offered to any interested patient. A standardized DOD/VA pain scale measured pain levels from 0 to 10 both pre- and immediately post-intervention with BFA. No personally identifiable information was collected and all data were deindentified.

A Wilcoxon signed-rank test was used to determine the statistical significance of changes in pain scores. 95\% confidence intervals were produced for all tests. Data was analyzed using SPSS.

\section{Results}

Twenty six patients meeting the inclusion criteria opted for BFA treatment over the course of the study period. Of these individuals, the majority (61.5\%) were men. Back conditions were the most common cause for pain (46\%), followed by headaches (19\%), joint pain (15\%), and neck conditions (12\%). Two individuals suffered from multiple conditions. Descriptive characteristics are displayed in Table 1.

Table 1: Subjects' Characteristics

\begin{tabular}{|c|c|}
\hline Age & N (\%) \\
\hline $18-24$ & $6(23)$ \\
\hline $25-29$ & $6(23)$ \\
\hline $30-34$ & $4(15)$ \\
\hline $35-39$ & $4(15)$ \\
\hline $40-44$ & $3(12)$ \\
\hline $45-49$ & $3(12)$ \\
\hline \multicolumn{2}{|c|}{ Gender } \\
\hline Female & $10(38)$ \\
\hline Male & $16(62)$ \\
\hline History of chronic neural and musculoskeletal condition \\
\hline Low Back & $12(46)$ \\
\hline Joint & $4(15)$ \\
\hline Neck & $3(12)$ \\
\hline Headache & $5(19)$ \\
\hline More than One Condition & $2(8)$ \\
\hline
\end{tabular}

A decrease in pain with the use of BFA was common; overall, 21 individuals (81\%) experienced a decrease in pain after the first administration of BFA as compared to 5 (19\%) who experienced no change. None of the 26 individuals experienced an increase in pain following BFA.

Table 2: Response Rates for First BFA Visit Only

\begin{tabular}{|c|c|c|}
\hline & Number of Patients & Percent of Patients \\
\hline Decrease in Pain & 21 & $81 \%$ \\
\hline No Change & 5 & $19 \%$ \\
\hline Increase in Pain & 0 & $0 \%$ \\
\hline Total & 26 & --- \\
\hline
\end{tabular}

The mean self-reported pain score before BFA was 5.19; the mean after BFA was 2.88. This correlates to a decrease in pain by 2.31 .

Table 3: Pain Before and Pain After BFA Administration

\begin{tabular}{|c|c|}
\hline & Mean (n=26) \\
\hline Pain Before (SD) & $5.1923(2.07883)$ \\
\hline Pain After (SD) & $2.8846(2.62796)$ \\
\hline Change in Pain (SD) & $-2.3077(1.71509)$ \\
\hline $\mathrm{p}<0.001$
\end{tabular}

For those with a baseline pain of 6 or less, the change in pain was slightly larger than for those with a baseline pain greater than 6, at a decrease of 2.41 versus 2.11 .

Table 4: Outcomes Associated with Baseline Pain Levels Before and After BFA Administration

\begin{tabular}{|c|c|c|}
\hline & $\begin{array}{c}\text { Baseline Pain } \leq \mathbf{6} \\
(\mathbf{n = 1 7 )}\end{array}$ & $\begin{array}{c}\text { Baseline Pain }>\mathbf{6} \\
(\mathbf{n}=\mathbf{9})\end{array}$ \\
\hline Pain Before Mean (SD) & $4.0588(1.63824)$ & $7.3333(0.50000)$ \\
\hline Pain After Mean (SD) & $1.6471(1.76569)$ & $5.2222(2.43812)$ \\
\hline Change in Pain (SD) & $-2.4118(1.46026)$ & $-2.1111(2.20479)$ \\
\hline
\end{tabular}


When comparing based on medical condition, those with low back pain had the most significant change in pain before and after BFA at a decrease of 2.54. This is followed by musculoskeletal pain (2.13) and headaches (2.00). The category "musculoskeletal pain" consists of those patients with neck pain, joint pain (ex. hip, leg), and miscellaneous generalized pain. Those with more than one condition were categorized along with "musculoskeletal pain," as both parties experienced musculoskeletal pain exclusively.

Table 5: Outcomes Associated with Medical Condition

\begin{tabular}{|c|c|c|c|}
\hline & Headache n=5 & Low Back Pain n=13 & Musculoskeletal Pain n=8 \\
\hline Pain Before Mean (SD) & $5(3.16228)$ & $5.3077(1.79743)$ & $5.1250(2.03101)$ \\
\hline Pain After Mean (SD) & $3(3.16228)$ & $2.7692(2.68185)$ & $3(2.56348)$ \\
\hline Change in Pain (SD) & $-2(2.12132)$ & $-2.5385(1.98391)$ & $-2.1250(.99103)$ \\
\hline
\end{tabular}

\section{Discussion}

Determining the efficacy of BFA is of emerging importance as factors of adverse effects, the potential for extended use, and cost-effectiveness favor it over NSAIDs, opioids, and other medications used to manage pain (8). In our analysis of 26 patients, BFA was found to be very effective overall in reducing pain with more or less efficacy depending on the intensity of baseline pain. No increase in pain was reported, and the majority of individuals experienced a decrease in pain with BFA administration.

Because BFA was found slightly more effective for those with a baseline pain of 6 or less, it is possible that BFA may be recommended for those experiencing a lower level of pain. BFA may also prove more effective for those with lower back pain than other pain types, but due to the limitations of this study, further research is necessary to make this conclusion.

\section{Limitations}

The primary limitation of this study is its small sample size. Because of its retrospective and patient-oriented nature, assigning a larger number of patients to BFA treatment was not possible. This study is similarly limited by its nonrandomized nature, as patients had agency over whether they opted into BFA.

\section{Conclusion}

BFA can be considered a promising alternative to NSAIDs in reducing pain in military settings where individuals experience high levels of pain concurrent with the need for quick recovery times. BFA effectively reduces pain and can be considered a promising treatment option for pain reduction for military primary care providers. The authors recommend further study of BFA in comparison to other treatment options in order to build upon this increasingly important field.

\section{Acknowledgements}

The authors disclose no potential conflicts of interest, financial or otherwise. The authors disclose no financial support received. The authors disclose no federal or government endorsement. The research is exempt from institutional review board approval in accordance with 45 CFR part 46.101(b)(4).

\footnotetext{
About authors

Ihor Korol is a physician assistant serving on active duty in US Army.

Ksenia Strakhova is a registered dental hygienist.

Matheus Azevedo is an emergency medical technician serving on active duty in US Army.
}

\section{References}

1. Collinsworth KM, Goss DL. Battlefield Acupuncture and Physical Therapy Versus Physical Therapy Alone After Shoulder Surgery. Med Acupunct. 2019;31(4):228-238. doi:10.1089/acu.2019.1372

2. Han B, Compton WM, Blanco C, Crane E, Lee J, Jones CM. Prescription Opioid Use, Misuse, and Use Disorders in U.S. Adults: 2015 National Survey on Drug Use and Health. Ann Intern Med. 2017;167(5):293-301. doi:10.7326/M17-0865

3. Mallinson TE. A review of Ketorolac as a prehospital analgesic. Journal of Paramedic Practice. 2017;9(12):522-526. doi:10.12968/jpar.2017.9.12.522.

4. Federman DG, Zeliadt SB, Thomas ER, Carbone GF Jr, Taylor SL. Battlefield Acupuncture in the Veterans Health Administration: Effectiveness in Individual and Group Settings for Pain and Pain Comorbidities. Med Acupunct. 2018;30(5):273-278. doi:10.1089/acu.2018.1296

5. Niemtzow RC. Battlefield Acupuncture. Medical Acupuncture. doi:10.1089/acu.2007.0603. 2007;19(4):225-228.

6. Levy CE, Casler N, FitzGerald DB. Battlefield Acupuncture: An Emerging Method for Easing Pain. Am J Phys Med Rehabil. 2018;97(3):e18-e19. doi:10.1097/PHM.0000000000000766

7. Sherman L. Battlefield acupuncture improves veterans' pain and sleep. The Journal of Chinese Medicine. 2019;(119):70-71.

8. Walker PH, Pock A, Ling CG, Kwon KN, Vaughan M. Battlefield acupuncture: Opening the door for acupuncture in Department of Defense/Veteran's Administration health care. Nurs Outlook. 2016;64(5):491-498. doi:10.1016/j.outlook.2016.07.008

9. Yeh CH, Chiang YC, Hoffman SL, et al. Efficacy of auricular therapy for pain management: a systematic review and meta-analysis. Evid Based Complement Alternat Med. 2014;2014:934670. doi:10.1155/2014/934670

10. Niemtzow, Richard C., et al. "Battlefield Acupuncture in the U.S. Military: A Pain-Reduction Model for NATO.” Medical Acupuncture, 2015;27(5):344-348. doi:10.1089/acu.2014.1055.

11. Curtis KM, Tepper NK, Jatlaoui TC, et al. U.S. Medical Eligibility Criteria for Contraceptive Use, 2016. MMWR Recomm Rep. 2016;65(No. RR-3):1-104. doi:10.15585/mmwr.rr6503a1external icon. 